J. Dairy Sci. 97:2633-2645

http://dx.doi.org/10.3168/jds.2013-7738

(C) American Dairy Science Association ${ }^{\circledR}, 2014$.

\title{
Multilocus sequence typing of Lactococcus lactis from naturally fermented milk foods in ethnic minority areas of China
}

\author{
Haiyan Xu, ${ }^{1}$ Zhihong Sun, ${ }^{1}$ Wenjun Liu, Jie Yu, Yuqin Song, Qiang Lv, Jiachao Zhang, Yuyu Shao, \\ Bilige Menghe, and Heping Zhang ${ }^{2}$ \\ Key Laboratory of Dairy Biotechnology and Engineering, Ministry of Education, Inner Mongolia Agricultural University, Huhhot, Inner Mongolia \\ 010018, P. R. China
}

\begin{abstract}
To determine the genetic diversity and phylogenetic relationships among Lactococcus lactis isolates, 197 strains isolated from naturally homemade yogurt in 9 ethnic minority areas of 6 provinces of China were subjected to multilocus sequence typing (MLST). The MLST analysis was performed using internal fragment sequences of 12 housekeeping genes $(\operatorname{car} \mathrm{B}, \operatorname{clpX}, d n a \mathrm{~A}$, groEL, mur $\mathrm{C}, m u r \mathrm{E}, p e p \mathrm{~N}, p e p \mathrm{X}, p y r \mathrm{G}, r e c \mathrm{~A}, r p o \mathrm{~B}$, and pheS). Six (dnaA) to 8 (murC) different alleles were detected for these genes, which ranged from 33.62 $(c l p \mathrm{X})$ to $41.95 \%$ (recA) GC (guanine-cytosine) content. The nucleotide diversity $(\pi)$ ranged from 0.00362 (murE) to 0.08439 (carB). Despite this limited allelic diversity, the allele combinations of each strain revealed 72 different sequence types, which denoted significant genotypic diversity. The $d_{N} / d_{S}$ ratios (where $d_{S}$ is the number of synonymous substitutions per synonymous site, and $d_{N}$ is the number of nonsynonymous substitutions per nonsynonymous site) were lower than 1 , suggesting potential negative selection for these genes. The standardized index of association of the alleles $\left(I_{A}^{S}=\right.$ 0.3038) supported the clonality of Lc. lactis, but the presence of network structure revealed by the split decomposition analysis of the concatenated sequence was strong evidence for intraspecies recombination. Therefore, this suggests that recombination contributed to the evolution of Lc. lactis. A minimum spanning tree analysis of the 197 isolates identified 14 clonal complexes and 23 singletons. Phylogenetic trees were constructed based on the sequence types, using the minimum evolution algorithm, and on the concatenated sequence $(6,192 \mathrm{bp})$, using the unweighted pair-group method with arithmetic mean, and these trees indicated that the evolution of our Lc. lactis population was cor-
\end{abstract}

Received November 19, 2013.

Accepted January 17, 2014.

${ }^{1}$ These authors contributed equally to this work.

${ }^{2}$ Corresponding author: hepingdd@vip.sina.com. related with geographic origin. Taken together, our results demonstrated that MLST could provide a better understanding of Lc. lactis genome evolution, as well as useful information for future studies on global Lc. lactis structure and genetic evolution, which will lay the foundation for screening Lc. lactis as starter cultures in fermented dairy products.

Key words: traditional fermented milk food, Lactococcus lactis, multilocus sequence typing, housekeeping gene

\section{INTRODUTION}

Lactococcus lactis is a species of lactic acid bacteria (LAB) used in dairy starter cultures, especially hard and semihard cheeses. This species is composed of 4 known subspecies: Lc. lactis ssp. cremoris, Lc. lactis ssp. lactis, Lc. lactis ssp. hordniae, and Lc. lactis ssp. tructae (Schleifer, 1987; Pérez et al., 2011). Lactococcus lactis ssp. hordniae isolated from the leafhopper Hordnia circellata has not been detected in dairy products (Schleifer et al., 1985), and Lc. lactis ssp. tructae was isolated from the intestinal mucus of brown trout ( Salmo trutta) and rainbow trout (Oncorhynchus mykiss) species (Pérez et al., 2011). Lactococcus lactis ssp. lactis is found in a variety of environments, including animal sources, dairy products, and plant surfaces (Klijn et al., 1995; Nomura et al., 2006), whereas Lc. lactis ssp. cremoris is isolated primarily from raw milk and other dairy products (Urbach et al., 1997; Nomura et al., 2006). Lactococcus lactis ssp. lactis and ssp. cremoris are widely used for industry and research and play a key role in the determination of shelf life, preservation, and organoleptic quality, thereby influencing the quality and safety of these fermented products (Smit et al., 2005). In addition, many strains of Lc. lactis ssp. lactis and ssp. cremoris carry plasmids encoding important traits, such as lactose catabolism, citrate utilization, proteinase production, bacteriocin production and immunity, bacteriophage resistance, exopolysaccharide production, as well as heavy metal resistance (McKay, 1983; Davidson et al., 1996). These broad applications 
might be attributed to their remarkable ecological adaptability to diverse habitats.

Lactococcus lactis ssp. lactis and ssp. cremoris differ by no more than $0.7 \%$ in their $16 \mathrm{~S}$ rDNA sequences (Salama et al.,1991) but display an average of only $85 \%$ DNA identity at the genome level (Wegmann et al., 2007). Some strains of Lc. lactis showing an Lc. lactis ssp. lactis phenotype according to classical distinction criteria, show an Lc. lactis ssp. cremoris genotype (cremoris genotype; Jarvis and Jarvis, 1981), meanwhile, phenotypic Lc. lactis ssp. cremoris showing an Lc. lactis ssp. lactis genotype (lactis genotype) have also been reported (Kelly et al., 2010; Tanigawa et al., 2010). Therefore, Lc. lactis has an unusual structure with 2 phenotypically distinct groups, Lc. lactis ssp. lactis and Lc. lactis ssp. cremoris, which may differ in genotype. Consequently, it has been difficult to accurately distinguish the 2 subspecies. Numerous methods, including DNA-DNA hybridization, small subunit rRNA gene sequencing, and PCR fingerprint analyses (Salama et al., 1991; Erlandson and Batt, 1997; Mangin et al., 1999; $\mathrm{Pu}$ et al., 2002), have been used for identification of Lc. lactis subspecies. However, their resolutions were low and the resulting data proved difficult for public database curation.

Multilocus sequence typing (MLST) is considered the gold standard for characterization of bacterial isolates and is based on the partial nucleotide sequences of multiple housekeeping genes (Maiden, et al., 1998; Urwin and Maiden, 2003; Aanensen and Spratt, 2005). It was recently shown to be a powerful technique for bacterial typing (Enright and Spratt, 1999), providing critical information for evolutionary history, population structure, and long-term epidemiology of bacterial species (Maiden, 2006; Turner and Feil, 2007). Although MLST has been used principally to study major bacterial pathogens, several recent MLST schemes were developed for LAB species, including Oenococcus oeni (de las Rivas et al., 2004), Lactobacillus plantarum (de las Rivas et al., 2006), Lactobacillus casei (Cai et al., 2007), Streptococcus thermophilus (Delorme et al., 2010), and Lactobacillus sanfranciscensis (Picozzi et al., 2010).

We examined the diversity and relationships of $197 \mathrm{Lc}$. lactis strains isolated from traditional fermented dairy products in different regions of China by characterizing population structure using an MLST scheme. The main aims of the present study were to apply MLST to assess phylogenetic relationship and evolutionary characteristics of these isolates and further reexamine the subspecies composition of Lc. lactis, which will contribute to screen starter cultures in the future.

\section{MATERIALS AND METHODS}

\section{Bacterial Strains and Genomic DNA Extraction}

A total of 197 Lc. lactis strains were selected from Collection Centre of Lactic Acid Bacteria of Inner Mongolia Agriculture University in China. Those included strains isolated from naturally fermented yogurt made by the Mongolian peoples in Chifeng, Xilin Gol, Hulunbeir, Bayan Nur of Inner Mongolia, by the Bai peoples in Dali of YunNan, and by the Tibetan peoples in Qinghai, Szechwan, Gansu, and Tibet of China from 2005 to 2009. Other than that, all strains had been achieved by $16 \mathrm{~S}$ rDNA sequences. Details information of strains are listed in Table 1.

Lactococcus lactis strains were maintained in M17broth (Oxoid, CM0817B, Wesel, Germany) supplemented with $5.0 \mathrm{~g} / \mathrm{L}(\mathrm{wt} / \mathrm{vol})$ of lactose at $30^{\circ} \mathrm{C}$ for 18 to $24 \mathrm{~h}$, and then strains were harvested by centrifugation and cell pellets were used for DNA extraction. Total genomic DNA was extracted from cultures by using a previously reported method (Yu et al., 2012). Purified DNA was diluted to a final concentration of $100 \mathrm{ng} / \mu \mathrm{L}$ for application.

\section{DNA Amplification and Sequencing for MLST}

The MLST analyses traditionally focus on allelic diversity of housekeeping genes, which match admitted criteria, including their presence as a single copy in all strains, their conserved sequence, their wide distribution across the chromosome, and their mutually unlinked location. The final selection included 12 housekeeping genes: dna $\mathrm{A}, \operatorname{pyr} \mathrm{G}, r p o \mathrm{~B}$, gro $\mathrm{EL}, r e c \mathrm{~A}$, $c l p \mathrm{X}, \operatorname{car} \mathrm{B}, m u r \mathrm{C}, p e p \mathrm{~N}, p e p \mathrm{X}, m u r \mathrm{E}$, and pheS. In addition, pyr $\mathrm{G}$, groEL, and recA were selected based on the results of a previous study on Lactobacillus delbrueckii (Kana and Watanabe, 2011), and pepN, pepX, and pheS were selected based on a study with Lc. lactis (Rademaker et al., 2007). Selection of the remaining loci $(d n a \mathrm{~A}, r p o \mathrm{~B}, \operatorname{clp} \mathrm{X}, \operatorname{car} \mathrm{B}, \operatorname{mur} \mathrm{C}$, and $m u r \mathrm{E})$ was based on the presence of SNP between Lc. lactis ssp. lactis IL-1403 and Lc. lactis ssp. cremoris SK11. These SNP were identified using comparative genome microarrays. These primers of 12 housekeeping genes were designed by Premier 5.0 (Premier Biosoft International, Palo Alto, CA) based on the known genome of Lc. lactis ssp. lactis IL1403, and the information of primers is listed in Table 2.

For each strain, the genomic DNA was used as a template for PCR amplification of MLST loci on the automatic thermal cycler (PTC-200, MJ Research, 
Table 1. Bacterial strains used in this study (16S rDNA sequences results)

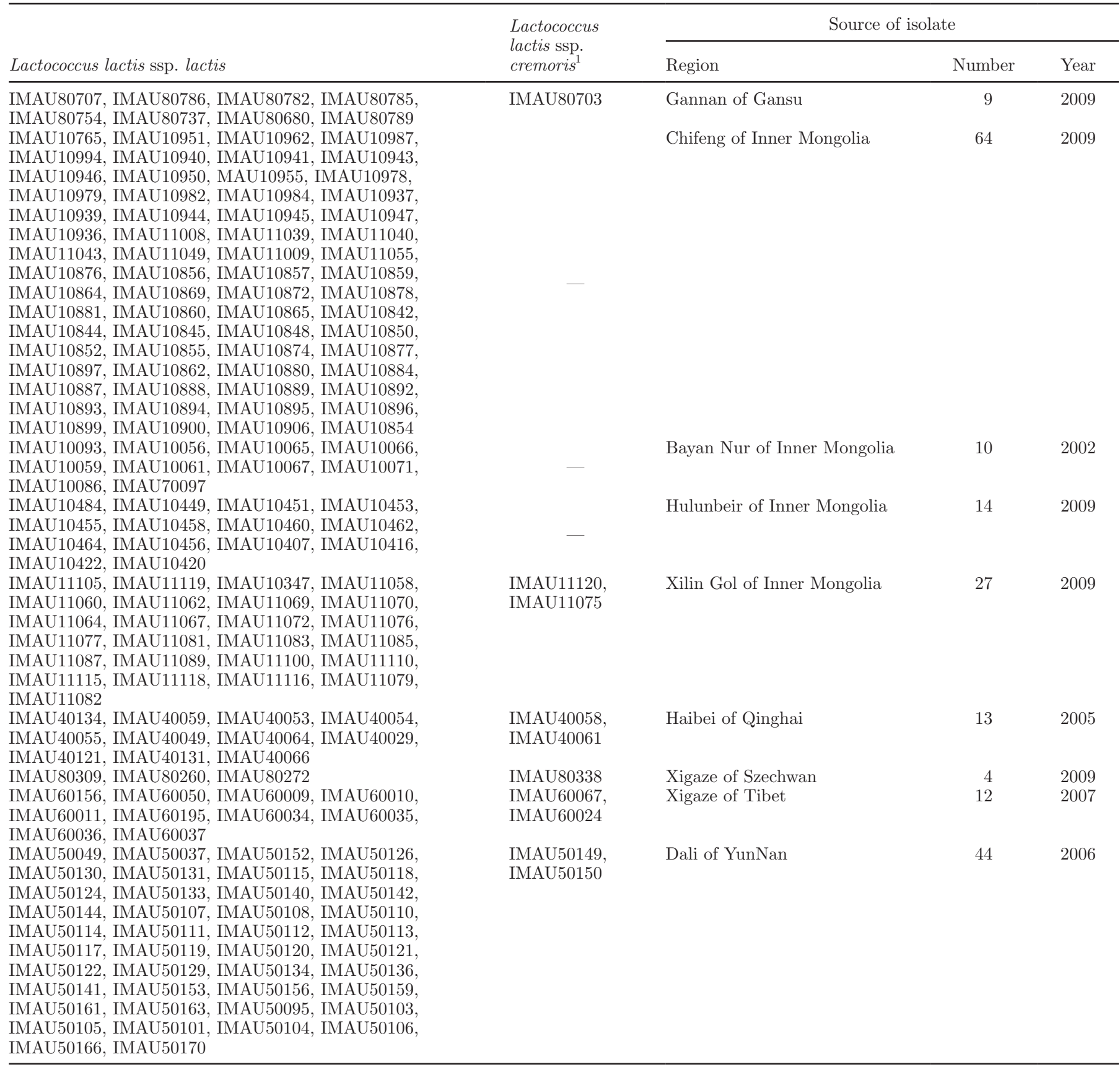

${ }^{1}$ Where no data is entered, no strains were used.

Waltham, MA). For each target, a PCR mixture (50 $\mu \mathrm{L})$ containing $150 \mathrm{ng}$ of genomic DNA, $10 \mathrm{~m} M$ deoxyribonucleotide, $10 \mathrm{pmol}$ of each primer, $2.5 \mathrm{U}$ of Taq polymerase (TransGen Biotech, Beijing City, China) in $1 \times$ PCR buffer (with $\mathrm{Mg}^{2+}$ ). The PCR amplification program consisted of an initial denaturation step at $94^{\circ} \mathrm{C}$ for $4 \mathrm{~min}$; 30 cycles of $94^{\circ} \mathrm{C}$ for $1 \mathrm{~min}, 50$ to $60^{\circ} \mathrm{C}$ (optimal annealing temperatures for each locus are listed in Table 2) for $45 \mathrm{~s}$, and $72^{\circ} \mathrm{C}$ for $1 \mathrm{~min}$; and a final extension step at $72^{\circ} \mathrm{C}$ for $7 \mathrm{~min}$. The PCR products were electrophoresed in a 1.2\% agarose gel. Sequencing of the PCR products was performed by Shanghai Majorbio Bio-pharm Technology Corporation (Shanghai, China). The same primers were used for PCR and sequencing on both DNA strands.

\section{Data Analysis for MLST}

For MLST analysis, forward and reverse sequences were trimmed, aligned, and analyzed using MEGA 5.0 
Table 2. Genes and primers used for multilocus sequence typing ${ }^{1}$

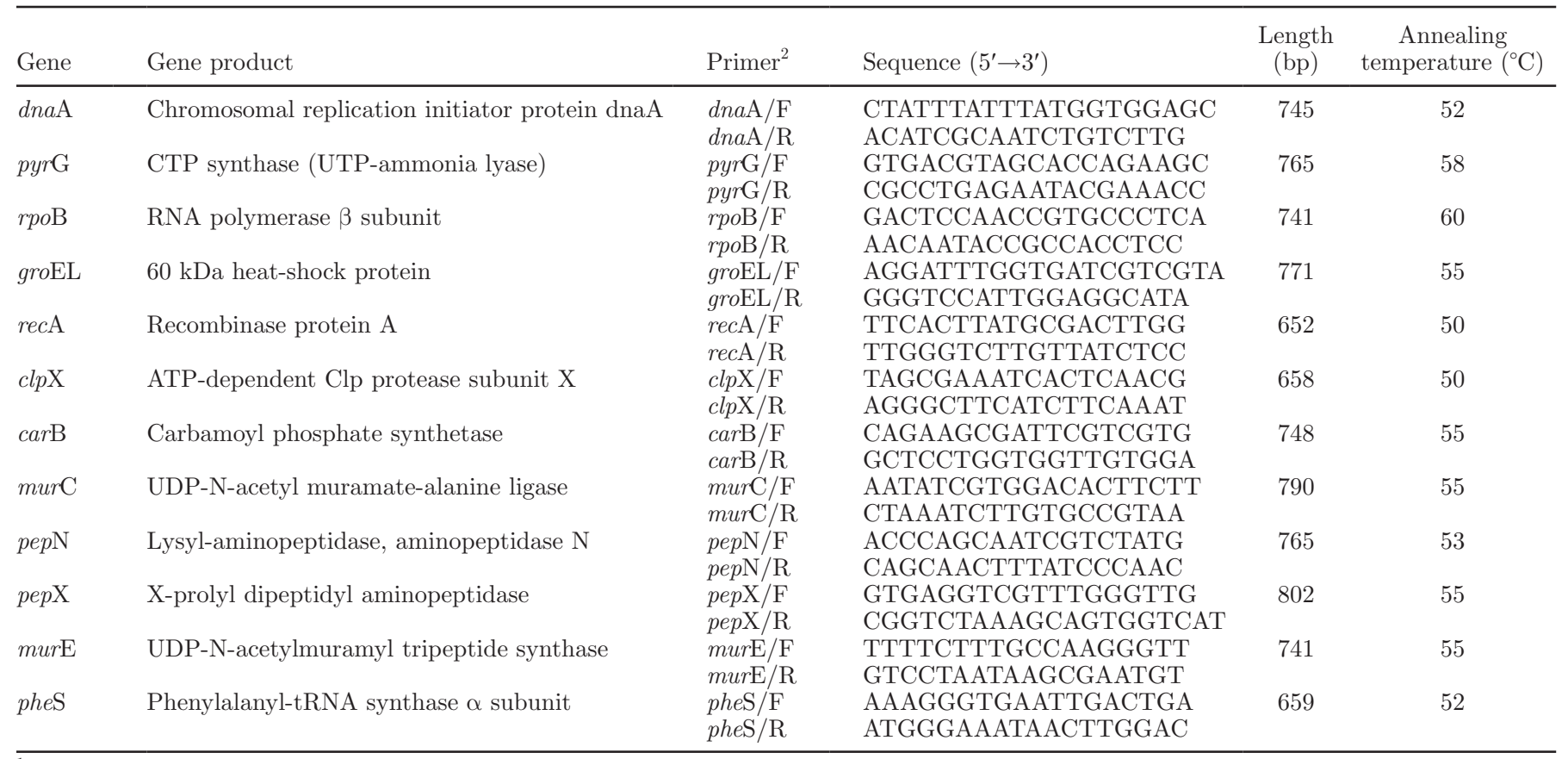

${ }^{1}$ Lactococcus lactis ssp. lactis IL1403 was used as the template for primer design.

${ }^{2} \mathrm{~F}=$ forward; $\mathrm{R}=$ reverse.

software package (version 5.0, www.megasoftware.net). Definition of alleles using a nonredundant data set and minimum spanning tree analysis were performed using the BioNumerics Software (version 6.0, Applied-Maths, Sint Maartens-Latem, Belgium). For each MLST locus, each different allele was assigned a different arbitrary number, and a unique combination of 12 allele numbers (allelic profile) unambiguously defined the sequence type (ST) of a strain. The same ST was used for several strains when they shared the same allelic profiles. Allelic profiles were used for subsequent analysis. Groups of isolates with closely related allelic profiles have been called clonal complexes (CC). The ST were grouped into CC with the BURST program developed by E. Feil (Feil et al., 2004) and located in the BioNumerics program.

The mean GC (guanine-cytosine) content of the DNA and the $d_{N} / d_{S}$ ratios (where $d_{S}$ is the number of synonymous substitutions per synonymous site, and $d_{N}$ is the number of nonsynonymous substitutions per nonsynonymous site) were calculated by using START 2.0 (Nei and Gojobori, 1986). This software was also used to calculate the index of association $\left(\boldsymbol{I}_{\boldsymbol{A}}\right)$ by the LIAN linkage analysis (Smith et al., 1993); nucleotide diversity $(\pi)$ per site was calculated using DnaSP version 5.0 (Rozas et al., 2003). The split decomposition method was used to assess the degree of tree-like structure for alleles of each locus and all ST using SplitsTree 4.0 (Huson, 1998; Huson and Bryant, 2006). The unweight- ed-pair group method using average linkages tree of ST was drawn with the START version 2.0 program (Jolley et al., 2001)

\section{Nucleotide Sequence Accession Numbers}

The sequences of the 12 MLST loci have been deposited in the GenBank database under accession numbers KF837137 to KF839500.

\section{RESULTS}

\section{Genetic Diversity}

We developed a new MLST scheme for 197 Lc. lactis strains based on the fragments of 12 housekeeping genes. By combining the 12 gene loci using the Bionumerics 6.0 software, MLST identified 72 different ST (Table 3 ), indicating high genotypic diversity. Of these ST, 51 corresponded to single isolates and 12 corresponded to 2 to 4 isolates. The best represented was ST5, identified in 38 isolates $(19.28 \%)$ of strains from as many as 6 regions across 4 provinces (Chifeng, Bayannur, and Xilin Gol of Inner Mongolia, Haibei of Qinghai, Gannan of Gansu, and Xigaze of Tibet), followed by ST13 (7.6\%), ST17 (5.58\%) and ST34 (5.58\%); the remaining ST accounted for less than $5 \%$ of isolates. Seven ST were associated with strains isolated from more than one region. In contrast, we found $65 \mathrm{ST}$ assigned to 
strains isolated from one specific region, as was the case for ST12, ST13, ST17, and ST19, which were identified only in strains from Chifeng, Inner Mongolia, and for ST31, ST34, and ST42, which were identified in strains collected from Dali, Yunnan.

The number of alleles per locus ranged from $6(d n a \mathrm{~A})$ to 18 (murC). The $\mathrm{GC}$ content of all alleles ranged from $33.62(\operatorname{clp} \mathrm{X})$ to $41.95 \%(r e c \mathrm{~A})$, consistent with the $38.42 \%$ GC content that has been observed across the entire Lc. lactis genome. The housekeeping genes examined by MLST had GC contents similar to those of Lc. lactis. ssp. lactis (strain IL1403), suggesting that these genes have been present in Lc. lactis for some time (Bolotin et al., 2001). The nucleotide diversity ( $\pi$, the average number of nucleotide differences per site between 2 randomly-selected isolates) ranged from 0.00362 (murE) to 0.08439 (carB; Table 4), which demonstrated the different evolution rates of 12 genes used in the new MLST scheme. The $d_{N}$ (number of nonsynonymous changes per nonsynonymous site) and $d_{S}$ (number of synonymous changes per synonymous site) values were calculated for each gene. The $d_{N} / d_{S}$ ratios were determined to be lower than 1 for all loci (Table 4 ), indicating the predominance of purifying selection preferentially associated with reducing AA variation.

\section{Recombination and Linkage Disequilibrium}

We measured intergenic recombination by estimating the linkage disequilibrium between the 12 loci using START2.0 (Nei and Gojobori, 1986). The $I_{A}$ (Smith et al., 1993) and standardized index of association $\left(I_{A}^{S}\right.$; Haubold and Hudson, 2000) are measures of the extent of linkage equilibrium within a population determined by quantifying the amount of recombination in a set of sequences and detecting association among alleles at different loci. If linkage equilibrium is evident due to frequent recombination events, the expected value of $I_{A}$ is zero. Clonal populations are identified by an $I_{A}$ value that differs significantly from zero. We calculated the $I_{A}$ and $I_{A}^{S}$ values to be 3.3423 and 0.3038 , respectively, for the 12 loci, indicating linkage disequilibrium or association among the alleles (Haubold and Hudson, 2000).

The method of split decomposition (Huson, 1998; Rozas et al., 2003) was used to assess the degree of tree-like structures present in the alleles at each locus in all 197 bacterial isolates. The algorithm used in this software can display conflicting results in the phylogenetic descent of sequences. A tree-like structure is created if the descent is clonal, whereas an interconnecting network resembling a parallelogram will appear wherever recombination has influenced the evolution of that gene (de las Rivas et al., 2006). The split graphs for all 12 loci showed different network structures, except for the murE gene (Figure 1a), suggesting that intragenic recombination occurred during the evolution of the other 11 loci. Yet, the parallelogram network of the 11 genes was not very apparent, indicating that intragenic recombination was not remarkable. A combined split graph based on a distance matrix comprising pairwise distances of all alleles of the 12 loci also revealed a network-like structure; the multiple parallel paths were indicative of incompatibilities resulting from recombination or recurrent mutation (Figure $1 \mathrm{~b}$ ).

\section{Phylogenetic Analysis}

To explore the relationships among the 197 strains, the population structure was analyzed using minimum spanning trees with an algorithm modified to take into account the number of mutations among allelic profiles (Bionumerics v. 6.0; Schouls et al., 2004). In this representation, strains with the same ST are grouped in one circle, the size of which is proportional to the number of strains. Assignment of ST to CC using BURST analysis (Feil et al., 2004) revealed that the 72 ST were divided among 14 CC (CC1 to CC14) and 23 singletons (Table 3), and CC can be defined as groups of profiles differing by no more than one gene from at least one other profile of the group. Strains within CC represented $88.3 \%$ of the total 197. In Figure 2, the shaded zones between certain groups of circles indicate that these profiles belong to the same CC. The most common CC was CC1 (pink zone), represented by 13 ST, and ST5 was most prevalent among the 197 strains, seen in $38(19.28 \%)$ total. Sequence type 5 had 7 single locus variants, ST18 had 6 single locus variants and 2 double-locus variants (Katherine et al., 2007), whereas ST6, ST23, and ST66 appeared to be closely related to $\mathrm{CC} 1$, as they differed from ST5 in only 2 loci. Sequence type 8, ST13, ST14, ST16, and ST19 were all isolated from strains in Chifeng of Inner Mongolia; ST22, ST23, ST25, ST26, ST27, and ST28 from Xilin Gol of Inner Mongolia; ST71 and ST72 from Gannan of Gansu; ST3 and ST6 from Bayannur of Inner Mongolia; and ST39 and ST66 were isolated from strains in Dali of Yunnan and in Aba of Szechwan, respectively (Figure 2).

All other CC comprised only 2 to 4 ST and a limited number of strains; in particular, those of the 4 branches of ST5 (Figure 2). From left to right, the first branch consisted of $\mathrm{CC} 3$ and $\mathrm{CC} 4$, which were connected by discontinuous links. The second branch consisted of CC6, CC7, and single ST; most strains with these ST were Lc. lactis ssp. cremoris, according to $16 \mathrm{~S}$ rRNA sequences, with the exception of ST56 and ST70, which identified as Lc. lactis ssp. lactis. Six $\mathrm{CC}$ (CC8, CC9, CC10, CC11, CC12, and CC14) and a few single ST were identified in the third branch, of 


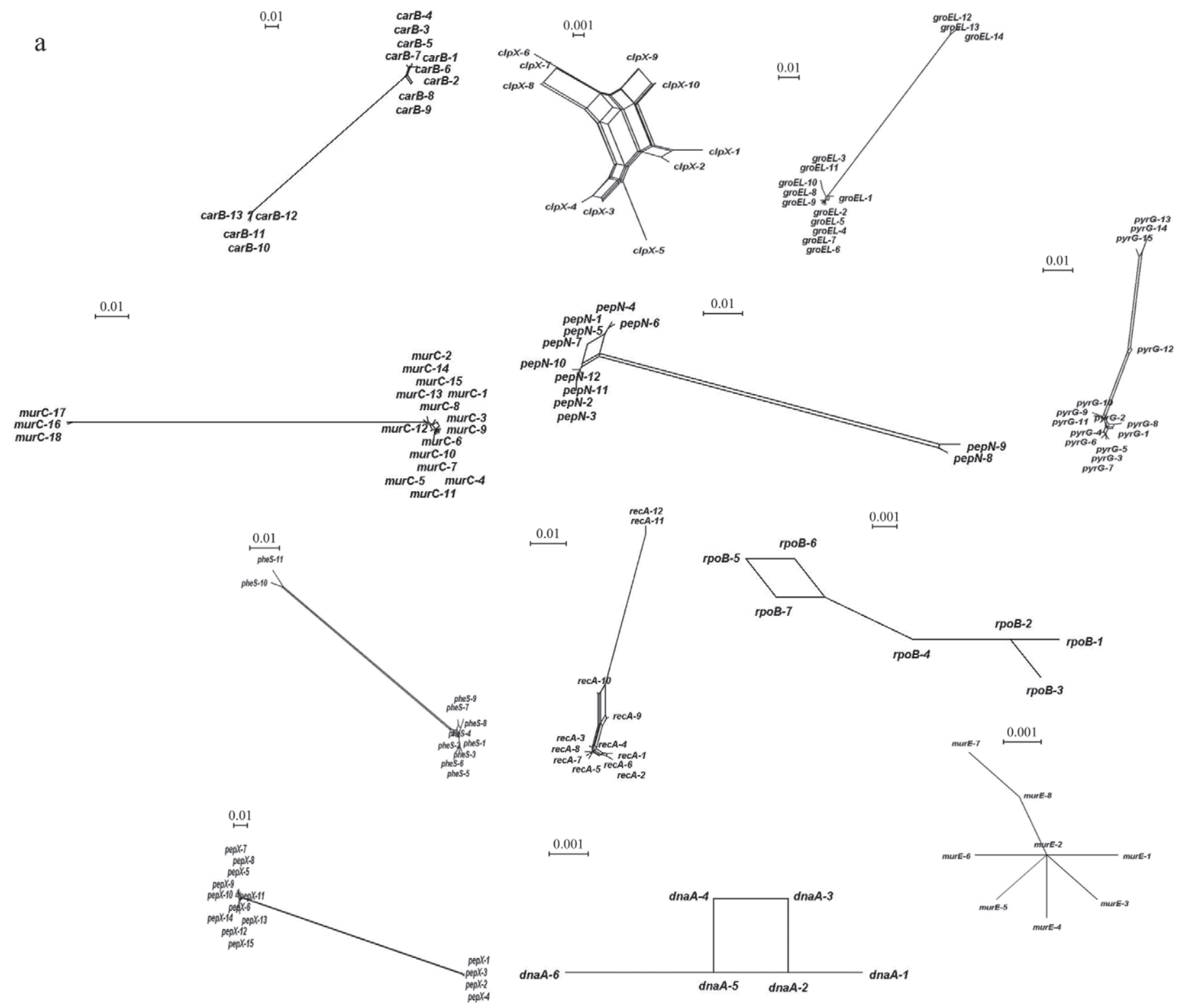

Figure 1. Split decomposition analysis based on the allelic profiles of the Lactococcus lactis strains. (a) Split decomposition of alleles for individual multilocus sequence typing loci and (b) combined split decomposition of alleles for all 12 multilocus sequence typing loci.

which all but CC9 were isolated from strains in Dali of Yunnan. The fourth branch comprised CC5 and CC13 with strains from Dali of Yunnan and Hulun Buir of Inner Mongolia.

We concatenated the 12 loci sequences, obtaining a much longer sequence alignment of $6,192 \mathrm{bp}$. This procedure is advantageous in that it can buffer local recombination consequences that potentially affect the recovery of true phylogenetic relationships among single or small numbers of genes (Hanage et al., 2005). An unweighted-pair group method using average linkages dendrogram of the concatenated sequence demonstrated the genetic relatedness among the Lc. lactis strains investigated in this study (Figure 3 ). In this tree, the 197 strains were separated into 2 major clusters, designated A and B. Most strains of cluster A belonged to Lc. lactis ssp. cremoris, except those of ST56 and ST70; whereas the strains of cluster B included $L c$. lactis ssp. lactis, with the exception of ST28. Cluster B was further divided into 2 subclusters. Cluster B1 consisted of one group representing ST of strains from Dali of Yunnan and Hulun Buir of Inner Mongolia and a second group representing the majority of strains from Xilin Gol and Chifeng of Inner Mongolia, Xigaze of Tibet, Haibei of Qinghai, and Gannan of Gansu; 4 strains from Bayannur of Inner Mongolia; and 2 strains from 


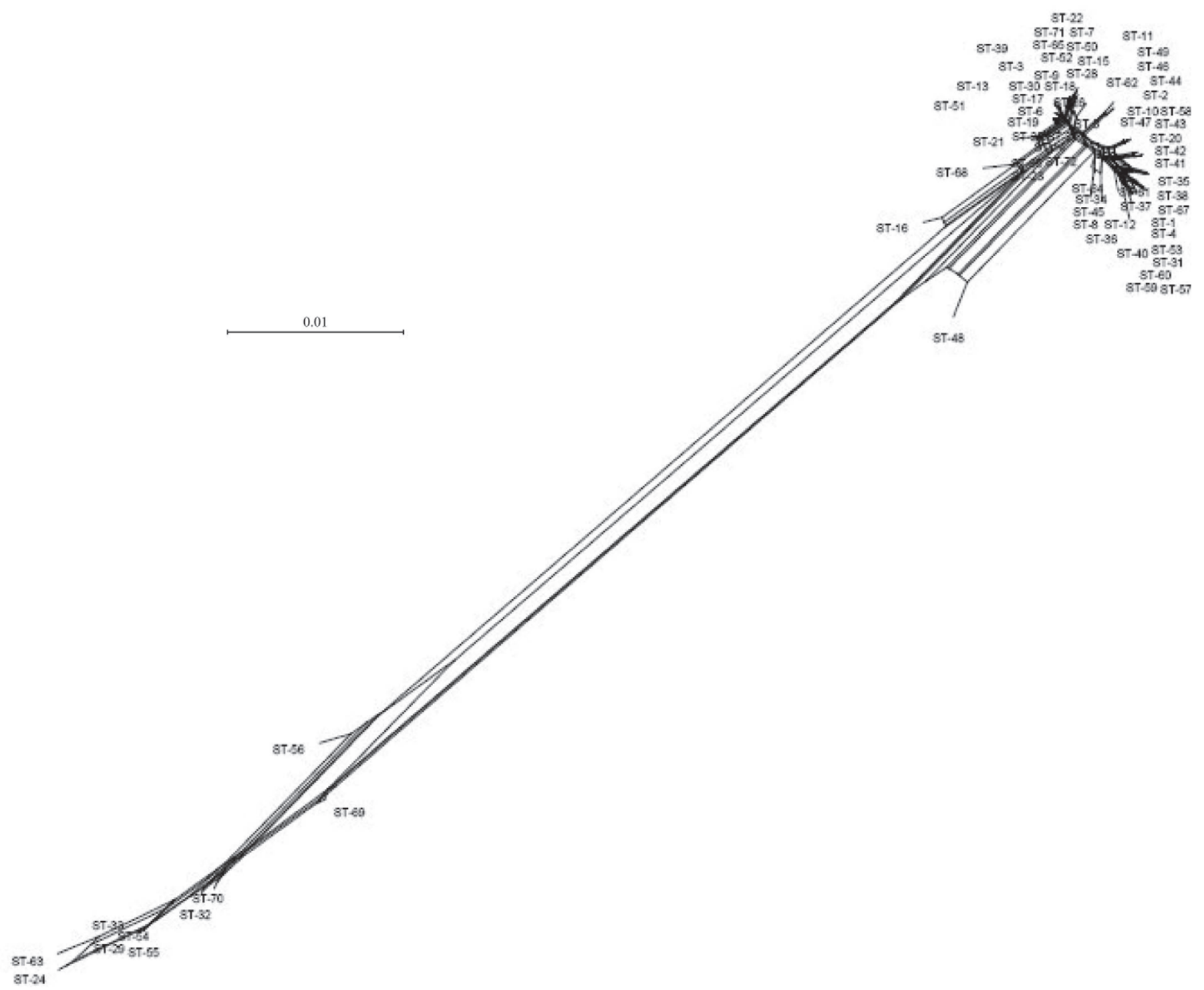

Figure 1 (Continued). Split decomposition analysis based on the allelic profiles of the Lactococcus lactis strains. (a) Split decomposition of alleles for individual multilocus sequence typing loci and (b) combined split decomposition of alleles for all 12 multilocus sequence typing loci.

Aba of Szechwan. Cluster B2 predominantly comprised strains from Dali of Yunnan, as well as 9 strains from Chifeng of Inner Mongolia, 6 from Bayannur of Inner Mongolia, and 1 from Aba of Szechwan.

\section{DISCUSSION}

A previous analysis of a collection of strains sampled from traditional fermented dairy products of a wide range of geographic locations, including 5 regions of Inner Mongolia, Tibet, Qinghai, Gansu, Szechwan, and Yunnan, revealed high genotypic diversity among $L c$. lactis. Multilocus sequence typing, which allows high- resolution analysis of molecular diversity, was used to evaluate the diversity and relationships of this species. However, few studies have characterized the genetic population diversity and evolutionary patterns of $L c$. lactis strains from different ecological origins. To contribute to the characterization of the Lc. lactis population structure, we designed an MLST typing scheme to examine 197 strains isolated from different geographical areas.

Multilocus sequence typing is considered the gold standard for characterizing isolates of bacterial species using the DNA sequences of selected housekeeping gene internal fragments (Urwin and Maiden, 2003). Most 
Table 3. Allelic profiles of analyzed Lactococcus lactis strains

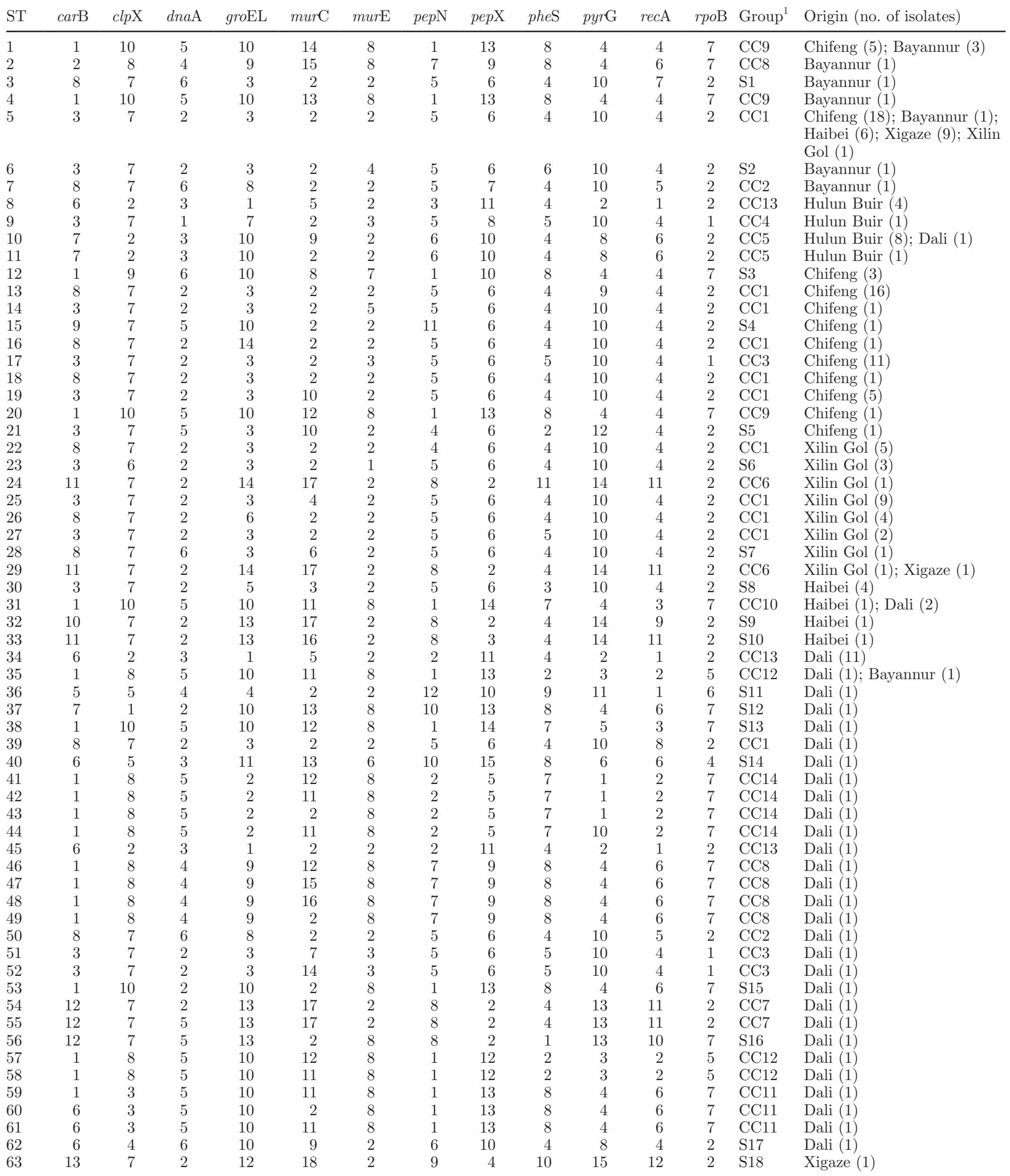

(Continued) 
Table 3 (Continued). Allelic profiles of analyzed Lactococcus lactis strains

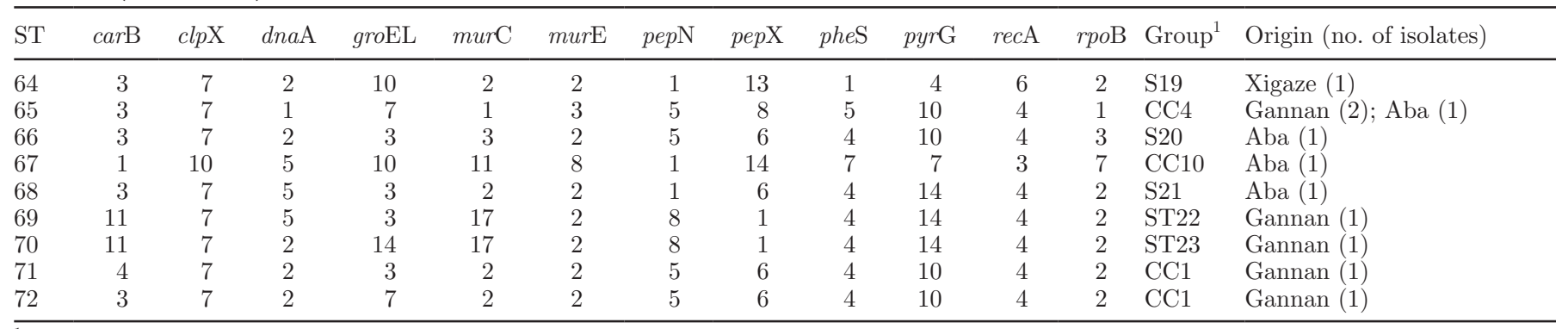

${ }^{1} \mathrm{CC}=$ clonal complex; $\mathrm{S}=$ singleton.

MLST schemes are based on 5 to 8 housekeeping genes with 450- to 500-bp fragments of each gene; however, small numbers of gene loci and short fragment lengths may decrease the amount of information obtained and the reliability of the results. In our study, 12 gene targets $(c a r \mathrm{~B}, \operatorname{clp} \mathrm{X}, d n a \mathrm{~A}, \operatorname{mur} \mathrm{C}, \operatorname{mur} \mathrm{E}, p e p \mathrm{~N}, p e p \mathrm{X}$, pyr $\mathrm{G}, \operatorname{rec} \mathrm{A}$, gro $\mathrm{EL}, u v r \mathrm{C}$, and $r p o \mathrm{~B})$ that gave reliable amplification products were used for MLST analysis. By combining the 12 gene loci, $72 \mathrm{ST}$ were identified with an average of 9 distinct alleles per locus, indicating a high level of genetic diversity within slowly evolving loci. A similar finding for Lc. lactis was obtained using MLST in previous studies (Delphine et al., 2010). Such differences are partly due to the number of isolates studied and the MLST scheme. The abundance of MLST loci and isolates of a wide geographical range contributed to the high genetic diversity.

The nucleotide diversity per site $(\pi)$ of the 12 genes varied from 0.00362 in murE to 0.08439 in carB. These values confirmed the differences in evolutionary rates among the genes used in this MLST scheme. The $\pi$ values were greater than 0.01 for 9 of the 12 loci, in which cases the variability was strongly correlated with the overall genomic variability. In comparison with other $\mathrm{LAB}$, the allelic diversity values for $L b$. plantarum (de las Rivas et al., 2006), Lb. delbruecki (Tanigawa and
Watanabe, 2011), and Oenococcus oeni (de las Rivas et al., 2004) ranged from 0.0004 to $0.0072,0.0051$ to 0.0096 , and 0.0011 to 0.0370 , respectively. Higher values were obtained for most of the species with available MLST data. Therefore, our Lc. lactis strain population appeared to encompass higher nucleotide diversity relative to these other LAB species, likely attributed to 2 Lc. lactis subspecies, which is similar to a report from 2010 (Delphine et al., 2010). The synonymous and nonsynonymous changes in the allelic sequences of a locus can provide information about the degree of selection exerted on a population. The $d_{N} / d_{S}$ ratios for 12 loci were $<1$, indicating a strong purifying selection against changes in AA composition, which is typical of housekeeping genes and desirable in MLST schemes (Sneath and Sokal, 1973).

Multilocus linkage disequilibrium was analyzed using $I_{A}$ and $I_{A}^{S}$ based on the MLST profile. High values are indicative of a strong clonal population. Thus, the analyzed genes in Lc. lactis appear far from linkage equilibrium according to our results. The $I_{A}^{S}(0.3038)$ value suggested strong clonal population, confirming that recombination played a key role in allelic distribution, but not frequently enough to prevent the emergence of clones. Split decomposition also provided strong evidence for intraspecies recombination according to the

Table 4. Allelic variation in 12 housekeeping genes

\begin{tabular}{lcccccc}
\hline Locus & Allele & $\begin{array}{c}\text { GC content } \\
(\%)\end{array}$ & $\pi^{1}$ & $d_{N}$ & $d_{S}$ & $d_{N} / d_{S}{ }^{2}$ \\
\hline carB & 13 & 36.72 & 0.08439 & 0.0199 & 0.6830 & 0.0291 \\
clpX & 10 & 33.62 & 0.01443 & 0.0006 & 0.0680 & 0.0090 \\
dnaA & 6 & 37.35 & 0.00406 & 0.0000 & 0.0181 & 0.0000 \\
groEL & 14 & 40.13 & 0.04173 & 0.0055 & 0.2092 & 0.0264 \\
murC & 18 & 37.81 & 0.03524 & 0.0038 & 0.2158 & 0.0176 \\
murE & 8 & 35.38 & 0.00362 & 0.0018 & 0.0076 & 0.2436 \\
pepN & 12 & 37.66 & 0.03757 & 0.0108 & 0.1747 & 0.0618 \\
pepX & 15 & 40.44 & 0.07644 & 0.0287 & 0.3824 & 0.0751 \\
pheS & 11 & 39.16 & 0.03009 & 0.0023 & 0.1762 & 0.0132 \\
pyrG & 15 & 41.95 & 0.03328 & 0.0052 & 0.1468 & 0.0355 \\
recA & 12 & 41.03 & 0.02580 & 0.0005 & 0.1219 & 0.0041 \\
rpoB & 7 & 39.81 & 0.00767 & 0.0008 & 0.0306 & 0.0249 \\
\hline
\end{tabular}

${ }^{1}$ Mean pairwise nucleotide differences per site.

${ }^{2} d_{N} / \mathrm{d}_{S}=$ the ratio of nonsynonymous to synonymous substitutions. 
64 Chifeng of Inner Mongolia

\section{Dali of Yunnan}

27 Xinlin Gol of Inner Mongolia

14 Hunlun Buir of Inner Mongolia

13 Haibei of Qinghai

12 Xigaze of Tibet

10 Bayannur of Inner Mongolia

9 Gannan of Gansu

4 Aba of Szechwan

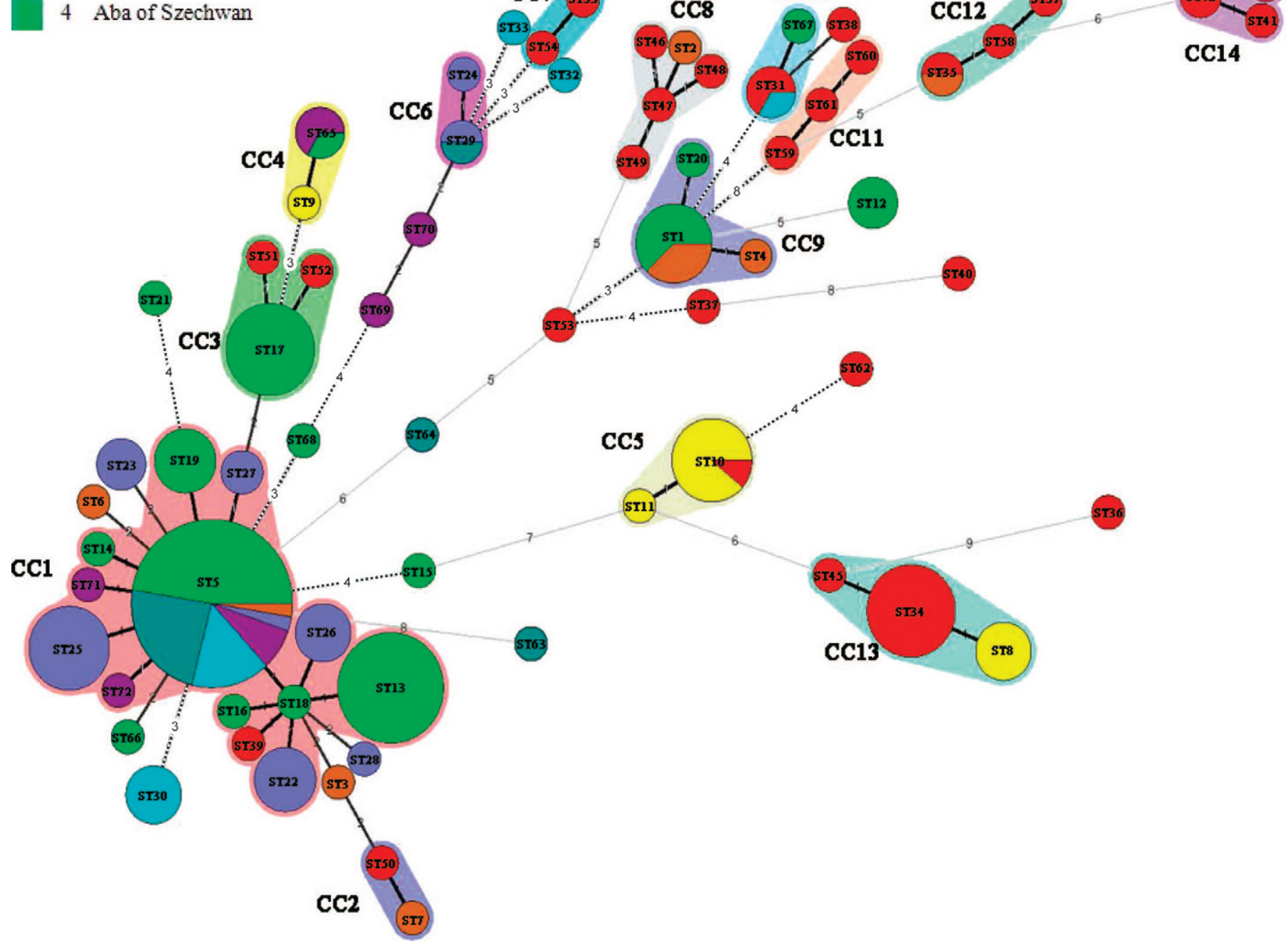

Figure 2. Minimum spanning tree analysis of the 197 Lactococcus lactis strains based on the allelic profiles of the 12 genes car $\mathrm{B}$, clpX, dnaA gro $\mathrm{EL}, m u r \mathrm{C}, \operatorname{mur} \mathrm{E}, p e p \mathrm{~N}, p h e S, p e p \mathrm{X}, p y r \mathrm{G}, r e c \mathrm{~A}$, and $r p o \mathrm{~B}$. Each circle corresponds to a sequence type (ST), and the circle size corresponds to the number of strains sharing the same ST. The shaded zones between certain groups of circles indicate that these profiles belong to the same clonal complex. Numerals connecting the circles indicate the number of allelic differences between the profiles. The strength of the link (bold, plain, or discontinuous) is correlated to the genetic similarity (number of common alleles) between profiles. CC $=$ clonal complex.

network structure. Split graphs for each locus showed tree-like or slight network-like structures, indicating that most of the genes were not significantly affected by intragenic recombination (Figure 1a). The concatenated sequence of the 12 MLST genes showed a network-like structure (Figure 1b), suggesting that the 12 gene fragments underwent widespread associative recombination overall. Therefore, our data suggest that recombination and selective pressure likely contributed to the evolution of Lc. lactis. 


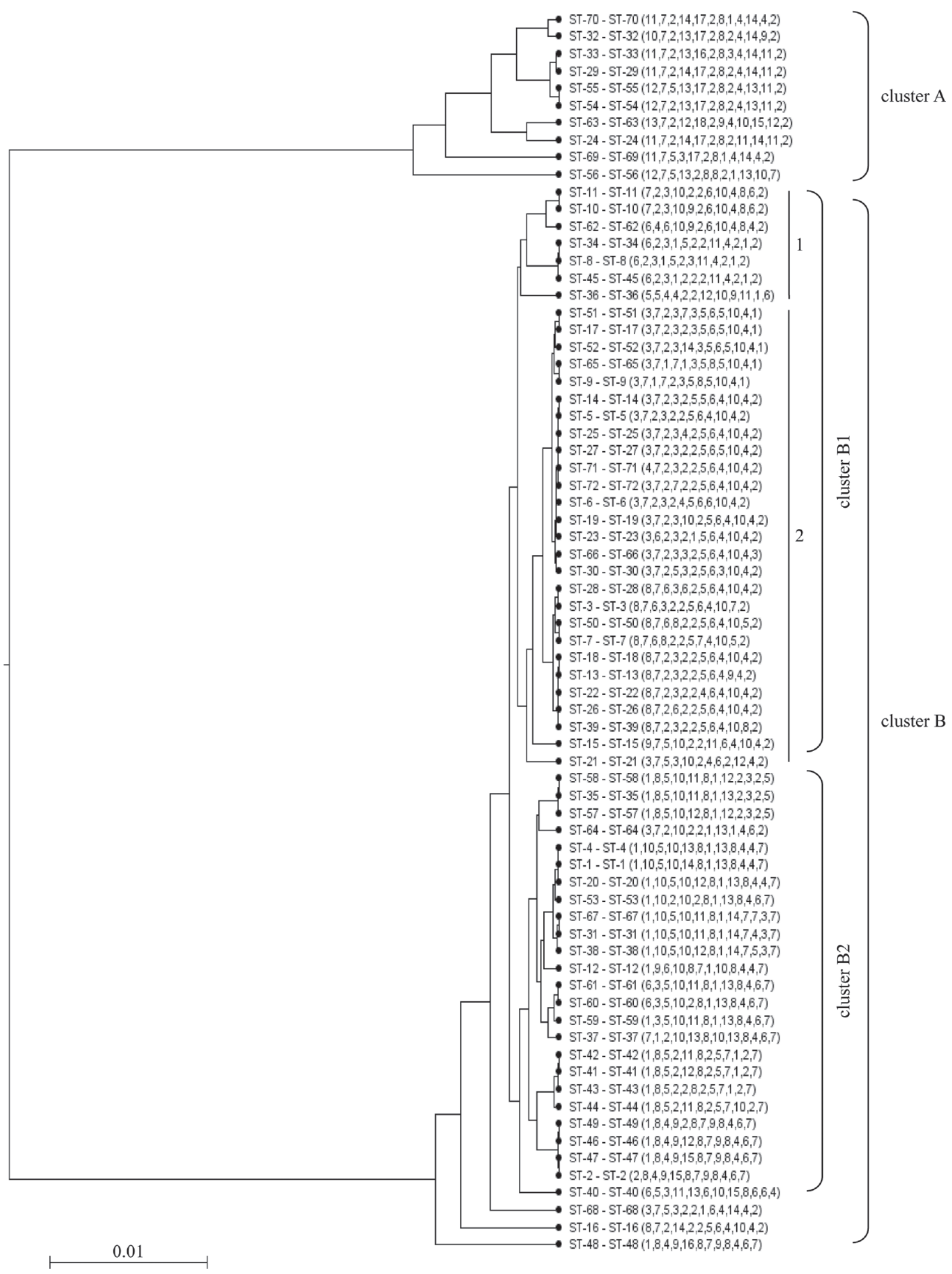

Figure 3. An unweighted pair-group method with arithmetic mean tree constructed from 72 concatenated nucleotide sequences of 12 multilocus sequence typing loci. 
According to minimum spanning tree and unweighted pair-group method with arithmetic mean tree analyses, IMAU50152 (ST56) and IMAU80707 (ST70) were assigned to Lc. lactis ssp. cremoris, which was classified as Lc. lactis ssp. lactis based on the $16 \mathrm{~S}$ rRNA sequence. Furthermore, IMAU80338 appeared to be distantly related to other cremoris strains due to the discontinuous link between ST68 and Lc. lactis ssp. cremoris according to the minimum spanning profile. In addition, the ST28 of the IMAU11116 strain was found in Lc. lactis ssp. cremoris, which clustered in the Lc. lactis ssp. lactis group. The $16 \mathrm{~S}$ rDNA gene sequences of lactis and cremoris subspecies differ by 9 to $10 \mathrm{bp}$ (depending on strain) within the first $200 \mathrm{bp}$ of the $16 \mathrm{~S}$ rDNA sequence, the difference is less than $1 \%$ for the total $16 \mathrm{~S}$ rDNA sequence of $1,500 \mathrm{bp}$. Therefore, $16 \mathrm{~S}$ rDNA sequence analysis is difficult to distinguish between lactis and cremoris subspecies (Salama et al., 1991). As early as 1991, Koehler et al. (1991) applied for traditional physiological and biochemical identification method and $16 \mathrm{~S}$ rDNA sequence analysis technology to identify Lc. lactis ssp. lactis and ssp. cremoris, but their results showed that Lc. lactis aren't accurately assigned to the subspecies level. This provides strong evidence for reclassification of Lc. lactis subspecies by MLST, consistent with previous reports supporting MLST as a new method for distinguishing subspecies (Rademaker et al., 2007; Kana and Watanabe, 2011).

Meanwhile, we explored the evolutionary relationships among Lc. lactis isolates from various regions of China. Based on our findings, CC1 originated from ST5, whose ancestral status suggests it is the most frequent ST in the population, and it was represented in 6 regions across 4 provinces (Feil, 2004). Interestingly, ST18 was only detected in a strain from Chifeng of Inner Mongolia, but it had 6 single locus variants (including ST5) and 2 double-locus variants, which is relatively primitive. In addition, 18 ST5 strains originated from Chifeng; thus, some of these isolates are likely to be ancestors to strains in this region of China. It was also confirmed that CC1 was represented in all regions except for Hulun Buir of Inner Mongolia. Moreover, abundant genetic diversity was observed in the isolates from Dali of Yunnan, which was apparent from the 44 strains that classified into $31 \mathrm{ST}$, and some isolates were related to the majority of those from Hulun Buir of Inner Mongolia. Our results differed from those obtained for similar species used in food fermentation, such as Lb. sanfranciscensis and $L b$. plantarum, in which no significant clusters were correlated with the geographic origin of the strains (Rozas et al., 2003; Picozzi et al., 2010). Similarly, Delphine et al. (2010) revealed little relationship between core and dispensable genome phylogenies by MLST, indicating that clonal diversification and phenotypic variability of the domesticated strains essentially arose through substantial genomic flux within the dispensable genome. The use of MLST will be of great help in defining the ecological and phylogenetic status of new lactococcal strains, and may be more informative than other genotyping methods (Delphine et al., 2010). As increasing numbers of isolates are collected from different regions, our findings enable comparisons with Lc. lactis isolates collected nationwide as well as providing information regarding the factors involved in the evolution of this important bacterium.

\section{CONCLUSIONS}

In conclusion, MLST was used to investigate the genetic polymorphisms and evolutionary relationships among $197 \mathrm{Lc}$. lactis isolates from various regions. First, an MLST scheme was developed for the study of Lc. lactis, which contributed data to the MLST database. Second, we demonstrated MLST to be an effective method for distinguishing Lc. lactis ssp. lactis and ssp. cremoris. Last, analysis of geographically diverse populations of bacterial isolates using MLST can provide a better understanding of Lc. lactis genomic evolution and useful information for further studies on global Lc. lactis structure and genetic evolution.

\section{ACKNOWLEDGMENTS}

This research was supported by National Natural Science Foundation of China (Beijing, China; Grant No. 31025019), Hi-Tech Research and Development Program of China (863 Planning, Beijing, China; Grant No.2011AA100902), Synergetic Innovation Center of Food Safety and Nutrition (Beijing, China), and the China Agriculture Research System (Beijing, China; Grant No.CARS-37).

\section{REFERENCES}

Aanensen, D. M., and B. G. Spratt. 2005. The multilocus sequence typing network: mlst.net. Nucleic Acids Res. 33:W728-733.

Bolotin, A., P. Wincker, S. Mauger, O. Jaillon, K. Malarme, J. Weissenbach, S. D. Ehrlich, and A. Sorokin. 2001. The complete genome sequence of the lactic acid bacterium Lactococcus lactis ssp. lactis IL1403. Genome Res. 11:731-753.

Cai, H., B. T. Rodríguez, W. Zhang, J. R. Broadbent, and J. L. Steele. 2007. Genotypic and phenotypic characterization of Lactobacillus casei strains isolated from different ecological niches suggests frequent recombination and niche specificity. Microbiology (Reading Engl.) 153:2655-2665.

Davidson, B. E., N. Kordias, M. Dobos, and A. J. Hillier. 1996. Genomic organization of lactic acid bacteria. Antonie van Leeuwenhoek 70:161-183.

de las Rivas, B., A. Marcobal, and R. Muñoz. 2004. Allelic diversity and population structure in Oenococcus oeni as determined from sequence analysis of housekeeping genes. Appl. Environ. Micro- 
biol. 70:7210-7219. http://dx.doi.org/10.1128/AEM.70.12.72107219.2004 .

de las Rivas, B., A. Marcobal, and R. Muñoz. 2006. Development of a multilocus sequence typing method for analysis of Lactobacillus plantarum strains. Microbiology 152:85-93.http://dx.doi. org $/ 10.1099 /$ mic. $0.28482-0$

Delorme, C., C. Bartholini, A. Bolotine, S. D. Ehrlich, and P. Renault. 2010. Emergence of a cell wall protease in the Streptococcus thermophilus population. Appl. Environ. Microbiol. 76:451-460.

Delphine, P., C. Beltramo, M. Coddeville, and P. Le Bourgeois. 2010. Genes but not genomes reveal bacterial domestication of Lactococcus Lactis. PLoS ONE 5:1-12.

Enright, M. C., and B. G. Spratt. 1999. Multilocus sequence typing. Trends Microbiol. 7:482-487.

Erlandson, K., and C. A. Batt. 1997. Strain-specific differentiation of lactococci in mixed starter culture populations using randomly amplified polymorphic DNA-derived probes. Appl. Environ. Microbiol. 63:2702-2707.

Feil, E. J. 2004. Small change: Keeping pace with microevolution. Nat. Rev. Microbiol. 2:483-495.

Feil, E. J., B. C. Li, D. M. Aanensen, W. P. Hanage, and B. G. Spratt. 2004. eBURST: Inferring patterns of evolutionary descent among clusters of related bacterial genotypes from multilocus sequence typing data. J. Bacteriol. 186:1518-1530.

Hanage, W. P., C. Fraser, and B. G. Spratt. 2005. Fuzzy species among recombinogenic bacteria. BMC Biol. 3:6.

Haubold, B., and R. R. Hudson. 2000. LIAN 3.0: Detecting linkage disequilibrium in multilocus data. Bioinformatics 16:847-848.

Huson, D. H. 1998. SplitsTree: Analyzing and visualizing evolutionary data. Bioinformatics 14:68-73.

Huson, D. H., and D. Bryant. 2006. Application of phylogenetic networks in evolutionary studies. Mol. Biol. Evol. 23:254-267.

Jarvis, A. W., and B. D. W. Jarvis. 1981. DNA homology among lactic streptococci. Appl. Environ. Microbiol. 41:77-83.

Jolley, K. A., E. J. Feil, M. S. Chan, and M. C. Maiden. 2001. Sequence type analysis and recombinational tests (START). Bioinformatics 17:1230-1231.

Kana, T., and K. Watanabe. 2011. Multilocus sequence typing reveals a novel subspeciation of Lactobacillus delbrueckii. Microbiology 157:727-738

Katherine, M. E. T., W. P. Hanage, C. Fraser, T. R. Connor, and B. G. Spratt. 2007. Assessing the reliability of eBURST using simulated populations with known ancestry. BMC Microbiol. 7:30. http://dx.doi.org/10.1186/1471-2180-7-30.

Kelly, W. J., L. J. H. Ward, and S. C. Leahy. 2010. Chromosomal diversity in Lactococcus lactis and the origin of dairy starter cultures. Genome Biol. Evol. 2:729-744

Klijn, N., A. H. Weerkamp, and W. M. de Vos. 1995. Detection and characterization of lactose-utilizing Lactococcus spp. in natural ecosystems. Appl. Environ. Microbiol. 61:788-792.

Koehler, G., W. Ludwig, and K. H. Schleifer. 1991. Differentiation of Lactococci by $16 \mathrm{~S}$ rRNA gene restriction analysis. FEMS Microbiol. Lett. 84:307-312.

Maiden, M. C. 2006. Multilocus sequence typing of bacteria. Annu. Rev. Microbiol. 60:561-588.

Maiden, M. C., J. J. A. Bygraves, E. Feil, G. Morelli, J. E. Russell, R. Urwin, Q. Zhang, J. Zhou, K. Zurth, D. A. Caugant, I. M. Feavers, M. Achtman, and B. G. Spratt. 1998. Multilocus sequence typing: A portable approach to the identification of clones within populations of pathogenic microorganisms. Proc. Natl. Acad. Sci. USA 95:3140-3145.

Mangin, I., D. Corroler, A. Reinhardt, and M. Gueguen. 1999. Genetic diversity among dairy lactococcal strains investigated by polymerase chain reaction with three arbitrary primers. J. Appl. Microbiol. 86:514-520.

McKay, L. L. 1983. Functional properties of plasmids in lactic streptococci. Antonie van Leeuwenhoek 49:259-274.

Nei, M., and T. Gojobori. 1986. Simple methods for estimating the numbers of synonymous and nonsynonymous nucleotide substitutions. Mol. Biol. Evol. 3:418-426.
Nomura, M., M. Kobayashi, T. Narita, H. Kimoto-Nira, and T. Okamoto. 2006. Phenotypic and molecular characterization of Lactococcus lactis from milk and plants. J. Appl. Microbiol. 101:396405.

Pérez, T., J. L. Balcázar, A. Peix, A. Valverde, E. Velázquez, I. de Blas, and I. Ruiz-Zarzuela. 2011. Lactococcus lactis ssp. tructae ssp. nov. isolated from the intestinal mucus of brown trout (Salmo trutta) and rainbow trout (Oncorhynchus mykiss). Int. J. Syst. Evol. Microbiol. 61:1894-1898.

Picozzi, C., G. Bonacina, I. Vigentini, and R. Foschino. 2010. Genetic diversity in Italian Lactobacillus sanfranciscensis strains assessed by multilocus sequence typing and pulsed-field gel electrophoresis analyses. Microbiology (Reading Engl.) 156:2035-2045.

Pu, Z. Y., M. Dobos, G. K. Limsowtin, and I. B. Powell. 2002. Integrated polymerase chain reaction-based procedures for the detection and identification of species and subspecies of the gram-positive bacterial genus Lactococcus. J. Appl. Microbiol. 93:353-361.

Rademaker, J. L., H. Herbet, M. J. Starrenburg, S. M. Naser, D. Gevers, W. J. Kelly, J. Hugenholtz, J. Swings, and J. E. van Hylckama Vlieg. 2007. Diversity analysis of dairy and nondairy Lactococcus lactis isolates, using a novel multilocus sequence analysis scheme and (GTG)5-PCR fingerprinting. Appl. Environ. Microbiol. 73:7128-7137.

Rozas, J., J. C. Sanchez-DelBarrio, X. Messeguer, and R. Rozas. 2003. DnaSP, DNA polymorphism analyses by the coalescent and other methods. Bioinformatics 19:2496-2497.

Salama, M., W. Sandine, and S. Giovannoni. 1991. Development and application of oligonucleotide probes for identification of Lactococcus lactis ssp. cremoris. Appl. Environ. Microbiol. 57:1313-1318.

Schleifer, K. H. 1987. Recent changes in the taxonomy of lactic acid bacteria. FEMS Microbiol. Rev. 46:201-203.

Schleifer, K. H., J. Kraus, C. Dvorak, R. Kilpper-Bälz, M. D. Collins, and W. Fischer. 1985. Transfer of Streptococcus lactis and related streptococci to the genus Lactococcus gen. nov. Syst. Appl. Microbiol. 6:183-195.

Schouls, L. M., H. G. van der Heide, L. Vauterin, P. Vauterin, and F. R. Mooi. 2004. Multiple-locus variable-number tandem repeat analysis of Dutch Bordetella pertussis strains reveals rapid genetic changes with clonal expansion during the late 1990s. J. Bacteriol. 186:5496-5505.

Smit, G., B. A. Smit, and W. J. Engels. 2005. Flavour formation by lactic acid bacteria and biochemical flavour profiling of cheese products. FEMS Microbiol. Rev. 29:591-610.

Smith, J. M., N. H. Smith, M. O'Rourke, and B. G. Spratt. 1993. How clonal are bacteria? Proc. Natl. Acad. Sci. USA 90:4384-4388.

Sneath, P. H. A., and R. R. Sokal. 1973. Numerical Taxonomy. W. H. Freeman \& Company, San Francisco, CA.

Tanigawa, K., H. Kawabata, and K. Watanabe. 2010. Identification and typing of Lactococcus lactis by matrix-assisted laser desorption ionization-time of flight mass spectrometry. Appl. Environ. Microbiol. 76:4055-4062.

Tanigawa, K., and K. Watanabe. 2011. Multilocus sequence typing reveals a novel subspeciation of Lactobacillus delbrueckii. Microbiology (Reading Engl.) 157:727-738.

Turner, K. M., and E. J. Feil. 2007. The secret life of the multilocus sequence type. Int. J. Antimicrob. Agents 29:129-135.

Urbach, E., B. Daniels, M. S. Salama, W. E. Sandine, and S. J. Giovannoni. 1997. The ldh phylogeny for environmental isolates of Lactococcus lactis is consistent with rRNA enotypes but not with phenotypes. Appl. Environ. Microbiol. 63:694-702.

Urwin, R., and M. C. Maiden. 2003. Multi-locus sequence typing: A tool for global epidemiology. Trends Microbiol. 11:479-487.

Wegmann, U., M. O'Connell-Motherway, A. Zomer, G. Buist, C. Shearman, C. Canchaya, M. Ventura, A. Goesmann, M. J. Gasson, O. P. Kuipers, D. van Sinderen, and J. Kok. 2007. Complete genome sequence of the prototype lactic acid bacterium Lactococcus lactis ssp. cremoris MG1363. J. Bacteriol. 189:3256-3270.

Yu, J., Z. Sun, W. Liu, Q. Bao, J. Zhang, and H. Zhang. 2012. Phylogenetic study of Lactobacillus acidophilus group, L. casei group and L. plantarum group based on partial hsp60, pheS and tuf gene sequences. Eur. Food Res. Technol. 234:927-934. 